\title{
Existence and Multiplicities of Solutions for Asymptotically Linear Ordinary Differential Equations Satisfying Sturm-Liouville BVPs with Resonance
}

\author{
Keqiang Li, Ronghua Tong \\ Department of Mathematics, Henan Normal University, Xinxiang, China \\ Email: likeqiang000@sina.com
}

How to cite this paper: Li, K.Q. and Tong, R.H. (2019) Existence and Multiplicities of Solutions for Asymptotically Linear Ordinary Differential Equations Satisfying Sturm-Liouville BVPs with Resonance. Journal of Applied Mathematics and Physics, 7, 1197-1211. https://doi.org/10.4236/jamp.2019.75080

Received: March 23, 2019

Accepted: May 26, 2019

Published: May 29, 2019

Copyright $\odot 2019$ by author(s) and Scientific Research Publishing Inc. This work is licensed under the Creative Commons Attribution International License (CC BY 4.0).

http://creativecommons.org/licenses/by/4.0/

\begin{abstract}
In this paper, we prove existence and multiplicities of solutions for asymptotically linear ordinary differential equations satisfying Sturm-Liouville boundary value conditions with resonance. Adding assumption $\mathrm{H}_{3}$ that is similar to (LL) in Theorem 1.1, by index theory and Morse theory, we obtain more nontrivial solutions.
\end{abstract}

\section{Keywords}

Asymptotically Linear Ordinary Differential Equations with Resonance, Multiple Solutions, Sturm-Liouville Boundary Value Problem, Index Theory, Morse Theory

\section{Introduction}

In this paper, we investigate the nontrivial solutions of asymptotically linear ordinary differential equations satisfying Sturm-Liouville BVPs with resonance. Various boundary value problems of asymptotically linear ordinary differential equations have been studied before. Most of them are gotten by the topological degree theory. There are also some papers about resonant problem. But the asymptotically linear ordinary differential equations with resonance aren't concerned ago. Here, we concern asymptotically linear ordinary differential equations satisfying both Sturm-Liouville boundary value and resonance. We solve the problem to get Theorem 1.1 in the following section.

Now, we consider solutions of the following Sturm-Liouville boundary value problem: 


$$
\begin{gathered}
x^{\prime \prime}(t)+f(t, x(t))=0, \\
x(0) \cos \alpha-x^{\prime}(0) \sin \alpha=0, \\
x(1) \cos \beta-x^{\prime}(1) \sin \beta=0,
\end{gathered}
$$

where $f \in C^{1}([0,1] \times \mathbf{R}, \mathbf{R}), 0 \leq \alpha<\pi, 0<\beta \leq \pi$. In this paper, $f^{\prime}$ denotes the derivative with respect to $x$. Our main result is the following theorem.

Theorem 1.1 Assume that $a_{0} \in H_{0}\left(1,(0,1), \frac{\pi}{2}, \frac{\pi}{2}\right)$ in $[1]$, i.e. $x^{\prime \prime}+a_{0}(t) x=0$ and (1.2)-(1.3) has one nontrivial solution $x=x_{0}(t)>0, \forall t \in[0,1]$, $\lambda \in L^{\infty}[0,1]$. $f$ satisfies the following two conditions:

$\left(\mathrm{H}_{1}\right) \quad a_{0}(t) \leq \frac{f(t, x)}{x}<\lambda(t) \quad$ as $\quad|x| \geq r>0, \quad r$ is a constant, $i(\lambda)=1$, $v(\lambda)=0, \quad i\left(a_{0}\right)=0, \quad v\left(a_{0}\right)=1$;

$\left(\mathrm{H}_{2}\right) \quad f(t, 0) \equiv 0,1 \bar{\epsilon}\left[i\left(\lambda_{0}\right), i\left(\lambda_{0}\right)+v\left(\lambda_{0}\right)\right]$, where $\lambda_{0}(t):=f^{\prime}(t, 0) ;$

For the sake of convenience, we denote $f_{1}(t, x)=f(t, x)-a_{0}(t) x$,

$\left(\mathrm{H}_{3}\right)$

$$
\int_{0}^{1} f_{1}(t,+\infty) x_{0} \mathrm{~d} t>0>\int_{0}^{1} f_{1}(t,-\infty) x_{0} \mathrm{~d} t,
$$

where $f_{1}(t,+\infty)=\liminf _{x \rightarrow+\infty} f_{1}(t, x), \quad f_{1}(t,-\infty)=\limsup _{x \rightarrow+\infty} f_{1}(t, x)$, and $x_{0}(t)(>0)$ is a nontrivial solution of $x^{\prime \prime}+a_{0}(t) x=0$ and $(1.2)-(1.3), \forall t \in[0,1]$. Then (1.1-1.3) has at least one nontrivial solution. Moreover, if we assume

$\left(\mathrm{H}_{4}\right) \quad v\left(\lambda_{0}\right)=0, i\left(\lambda_{0}\right) \geq 2$.

Then (1.1-1.3) has at least two nontrivial solutions.

In this paper, for any $a \in L^{\infty}[0,1], i(a)$ and $v(a)$ denote its index and nullity of the associated linear ordinary differential equation (see [2] [3] for reference). In Section 2, we will briefly recall the index and its properties. For the readers' convenience, we give an example: Assume $\lambda$ is a constant, $\alpha=\frac{\pi}{2}$ and $\beta=\frac{\pi}{2}$. Then

$$
i(\lambda)= \begin{cases}0 & \text { as } \lambda \leq 0, \\ k+1 & \text { as } \lambda \in\left(k^{2} \pi^{2},(k+1)^{2} \pi^{2}\right],\end{cases}
$$

and

$$
v(\lambda)= \begin{cases}0 & \text { as } \lambda \neq k^{2} \pi^{2}, \\ 1 & \text { as } \lambda=k^{2} \pi^{2}, k \in \mathbf{N}^{+} \bigcup\{0\} .\end{cases}
$$

In [2], an index for second order linear Hamiltonian systems was defined. And in [3], an index for more general linear self-adjoint operator equations was developed. In [4] [5] [6] [7], by Conley, Zehnder and Long, an index theory for sympletic paths was defined. More applications about these index theories can be found in [8]-[13]. As in [11], throughout this paper, for $a_{1}, a_{2} \in L^{\infty}[0,1]$, we write $a_{1} \leq a_{2}$, if $a_{2}(t)-a_{1}(t) \geq 0$, for a.e. $t \in[0,1]$; we write $a_{1}<a_{2}$, if $a_{1} \leq a_{2}$, and $a_{2}(t)-a_{1}(t)>0$ holds on a subset of $[0,1]$ with nonzero measure. 
It is well known [14] that under non-resonant conditions

$$
(2 k \pi)^{2}+\delta \leq \frac{f(t, x)}{x} \leq(2(k+1) \pi)^{2}-\delta, \text { as }|x|>r>0, k \in \mathbf{N},
$$

the existence of solutions of a second order nonlinear ordinary differential equation. Such conditions are called nonresonant. Resonant conditions in [15] [16]:

$$
(2 k \pi)^{2} \leq \frac{f(t, x)}{x} \leq(2(k+1) \pi)^{2}, \text { as }|x|>r>0, k \in \mathbf{N},
$$

are not enough for existence solutions of (1.1-1.2). An additional condition called the $(L L)$ condition like $\left(\mathrm{H}_{3}\right)$ is usually needed. For resonant conditions, we refer to [15] [16] [17]. These three papers [14] [15] [16] are about existence of solutions.

In [18], under resonance conditions, periodic solutions of nonlinear second order ordinary differential equations are considered. Second order Hamiltonian systems satisfying Sturm-Liouville boundary vale with the nonresanonce are considered in [3]. First order asymptotical linear Hamiltonian systems satisfying Sturm-Liouville boundary vale with the nonresanonce are studied in [19]. In [20] [21], $\alpha=0, \beta=0$, the existence of solutions of (1.1-1.3) is investigated.

In this paper, we study the existence of equations with resonance conditions. In order to prove our theorem, we construct the corresponding functional:

$$
\begin{aligned}
\varphi(x)= & \frac{1}{2}\left[\int_{0}^{1}\left|x^{\prime}(t)\right|^{2} \mathrm{~d} t-(x(1), x(1)) k(\beta)+(x(1), x(1)) k(\alpha)\right] \\
& -\int_{0}^{1} F(t, x(t)) \mathrm{d} t, \forall x \in E .
\end{aligned}
$$

where $F(t, u)=\int_{0}^{u} f(t, s) \mathrm{d} s, u \in \mathbf{R}, \quad k(t)=\cot t=\frac{\cos t}{\sin t}, k(t)=0$ as $t=0$ or $\pi$, and $E$ will be described in Section 2. This functional $\varphi(x)$ is continuous differentiable on $E$, and any critical point of $\varphi$ corresponds to a solution of (1.1)-(1.3).

In Section 3, we will give proofs by the Morse theory following [11] [17].

\section{Index Theory for Linear Duffing Equations}

For any $a \in L^{\infty}[0,1]$, consider the following equation:

$$
\left\{\begin{array}{l}
x^{\prime \prime}(t)+a(t) x=0, \\
x(0) \cos \alpha-x^{\prime}(0) \sin \alpha=0, \\
x(1) \cos \beta-x^{\prime}(1) \sin \beta=0,
\end{array}\right.
$$

where $0 \leq \alpha<\pi, 0<\beta \leq \pi$. Define a Hilbert space $E:=E_{\alpha, \beta}$. Here $E_{\alpha, \beta}=H^{1}[0,1]$ as $\alpha, \beta \in(0, \pi) ; \quad E_{0, \beta}=\left\{x \in H^{1}[0,1] \mid x(1)=0\right\}$ as $\beta \in(0, \pi)$; $E_{\alpha, 0}=\left\{x \in H^{1}[0,1] \mid x(0)=0\right\}$ as $\alpha \in(0, \pi)$ and

$E_{0, \pi}=\left\{x \in H^{1}[0,1] \mid x(1)=0=x(0)\right\}$. With norm $\|x\|_{E}:=\left\{\int_{0}^{1}|x(t)|^{2}+\left|x^{\prime}(t)\right|^{2} \mathrm{~d} t\right\}^{\frac{1}{2}}$ and a bilinear form as $q_{a}(\cdot, \cdot)$ as follows 


$$
\begin{aligned}
q_{a}(x, y)= & \int_{0}^{1}\left[\left|x^{\prime}(t)\right|^{2} \mathrm{~d} t-(x(1), x(1)) k(\beta)+(x(0), x(0)) k(\alpha)\right] \\
& -\int_{0}^{1} a(t) x(t) y(t) \mathrm{d} t, \forall x, y \in E .
\end{aligned}
$$

From proposition 2.1.1 and 2.3.3 in [3], we have the following properties.

Proposition 2.1 For any $a \in L^{\infty}[0,1]$,

1) The $E$ can be divided into three parts:

$$
E=E^{+}(a) \oplus E^{0}(a) \oplus E^{-}(a)
$$

such that $q_{a}$ is positive definite, null and negative definite on $E^{+}(a), E^{0}(a)$ and $E^{-}(a)$ respectively. Furthermore, $E^{0}(A)$ and $E^{-}(A)$ are finitely dimensional. We call $v(a):=\operatorname{dim} E^{0}(a)$ and $i(a):=\operatorname{dim} E^{-}(a)$ the nullity and index respectively.

2) $(i(a), v(a)) \in \mathbf{N} \times\{0,1\}$.

3) $v(a)$ is the dimension of the solution subspace of (2.1-2.3), and $i(a)=\sum_{s<0} v(a+s)$.

4) If $a_{1} \leq a_{2}$, then $i\left(a_{1}\right) \leq i\left(a_{2}\right)$ and $i\left(a_{1}\right)+v\left(a_{1}\right) \leq i\left(a_{2}\right)+v\left(a_{2}\right)$; if $a_{1}<a_{2}$, then $i\left(a_{1}\right)+v\left(a_{1}\right) \leq i\left(a_{2}\right)$.

5) There exists $\delta>0$ such that

$$
q_{a}(u, u) \geq \delta\|u\|_{E}^{2}, \forall u \in E^{+}(a) .
$$

Remarks: 1) The notation $\oplus$ means that the space $E$ is the direct sum of some subspaces.

2) By 4), we can see the index has monotonicity.

1) Let $a(t)=0, \alpha=\frac{\pi}{2}$, and $\beta=\frac{\pi}{2}$. Then (2.1) has a nontrivial solution $x=c(\in \mathbf{R}) \neq 0$. So $v(0)=1, i(0)=0$. If $a(t)=\pi^{2}, \alpha=\frac{\pi}{2}$, and $\beta=\frac{\pi}{2}$, then (2.1) has a nontrivial solution $c_{1} \sin \pi t, c_{1} \neq 0$. So $v\left(\pi^{2}\right)=1, i\left(\pi^{2}\right)=1$. If $a(t)=k^{2} \pi^{2}, \quad \alpha=\frac{\pi}{2}$, and $\beta=\frac{\pi}{2}$, then (2.1) has a nontrivial solution $c_{k 1} \sin k \pi t$, $c_{k 1} \neq 0$. So by Proposition $2.1(3)$, $i\left(k^{2} \pi^{2}\right)=\sum_{s<0} v\left(k^{2} \pi^{2}+s\right)=\sum_{n=1}^{k-1} v\left(n^{2} \pi^{2}\right)=1+(k-1)=k, \quad v\left(k^{2} \pi^{2}\right)=1$.

The following lemmas are useful for us to prove the results.

Lemma 2.2 The norm $\|x\|_{C}:=\sup _{0 \leq t \leq 1}|x(t)| \leq C_{*}\|x\|_{E}$, for any $x \in E$, where $C_{*}(\in \mathbf{R})$ is a positive constant.

Lemma 2.3 If $\left(\mathrm{H}_{1}\right)$ holds, then we have that $E=\operatorname{span}\left\{x_{0}\right\} \oplus E^{+}(\lambda)$, where $x_{0}$ is given in Theorem 1.1.

Proof By Proposition 2.1 (1) and conditions $v(\lambda)=0, i(\lambda)=1$, we have that

$$
E^{0}(\lambda)=\{\theta\}, \operatorname{dim} E^{-}(\lambda)=1
$$

By Proposition 2.1 (1) and (3), we know that with respect to $\lambda \in L^{\infty}[0,1]$, the following decomposition holds, 


$$
E=E^{-}(\lambda) \oplus E^{0}(\lambda) \oplus E^{+}(\lambda)=E^{-}(\lambda) \oplus E^{+}(\lambda) .
$$

Since $E^{-}(\lambda)$ is one dimensional space, we can assume that $\left\{e^{-}\right\}$is a base of $E^{-}(\lambda)$, i.e. $E^{-}(\lambda)=\operatorname{span}\left\{e^{-}\right\}$. So for any $x \in E$, we have

$$
x=x^{-}+x^{+}=c_{0} e^{-}+x^{+},
$$

where $x^{-} \in E^{-}(\lambda), \quad x^{+} \in E^{+}(\lambda)$, and $c_{0}$ is a constant. For $x_{0} \in E$, we have the decomposition $x_{0}=c_{1} e^{-}+e^{+}$, where $e^{+} \in E^{+}(\lambda)$ and $c_{1}$ is a constant. It is obvious that $c_{1} \neq 0$. Indeed, if $c_{1}=0$, then $x_{0}=e^{+}$. By definition of $q_{\lambda}(\cdot, \cdot)$, we will have a contradiction that

$$
\begin{aligned}
& q_{\lambda}\left(x_{0}, x_{0}\right)= \int_{0}^{1}\left(x_{0}^{\prime}, x_{0}^{\prime}\right) \mathrm{d} t-\left(x_{0}(1), x_{0}(1)\right) k(\beta) \\
&+\left(x_{0}(1), x_{0}(1)\right) k(\alpha)-\int_{0}^{1} \lambda(t) x_{0}^{2} \mathrm{~d} t \\
&= \int_{0}^{1}(a(t)-\lambda(t)) x_{0}^{2} \mathrm{~d} t<0 \\
& q_{\lambda}\left(e^{+}, e^{+}\right)=\int_{0}^{1}\left(e^{+}, e^{+}\right) \mathrm{d} t-\left(e^{+}(1), e^{+}(1)\right) k(\beta)+\left(e^{+}(0), e^{+}(0)\right) k(\alpha) . \text { So } \\
&-\int_{0}^{1} \lambda(t)\left(e^{+}, e^{+}\right) \mathrm{d} t \geq 0
\end{aligned}
$$

and

we obtain

$$
\begin{aligned}
x & =c_{0} e^{-}+x^{+} \\
& =\frac{c_{0}}{c_{1}}\left(c_{1} e^{-}+e^{+}\right)-\frac{c_{0}}{c_{1}} e^{+}+x^{+} \\
& =\frac{c_{0}}{c_{1}} x_{0}+\left(x^{+}-\frac{c_{0}}{c_{1}} e^{+}\right) .
\end{aligned}
$$

We have proved that $E=\operatorname{span}\left\{x_{0}\right\} \cup E^{+}(\lambda)$. It is also obvious that $\operatorname{span}\left\{x_{0}\right\} \cap E^{+}(\lambda)=\{\theta\}$. In fact that if $x(\neq \theta) \in \operatorname{span}\left\{x_{0}\right\} \cap E^{+}(\lambda)$, we have that on the one hand for $x \in \operatorname{span}\left\{x_{0}\right\}$. Then there exists a $c(\in \mathbf{R}) \neq 0$ such that $x=c x_{0}$ and

$$
\begin{aligned}
q_{\lambda}(x, x)= & \int_{0}^{1} c^{2}\left|x_{0}^{\prime}\right|^{2}-c^{2}\left(x_{0}(1), x_{0}(1)\right) k(\beta) \\
& +c^{2}\left(x_{0}(1), x_{0}(1)\right) k(\alpha)-\int_{0}^{1} c^{2} \lambda(t) x_{0}^{2} \mathrm{~d} t \\
= & \int_{0}^{1} c^{2}\left(a_{0}(t)-\lambda(t)\right) x_{0}^{2} \mathrm{~d} t<0 ;
\end{aligned}
$$

on the other hand for $x \in E^{+}(\lambda)$,

$$
\begin{aligned}
q_{\lambda}(x, x)= & \int_{0}^{1}\left|x^{\prime}(t)\right|^{2} \mathrm{~d} t-(x(1), x(1)) k(\beta)+(x(1), x(1)) k(\alpha) \\
& -\int_{0}^{1} \lambda(t) x^{2}(t) \mathrm{d} t \geq 0 .
\end{aligned}
$$

By Proposition 2.1 (1) and (2.2), we have $x=\theta$. This is a contradiction. So the proof is completed.

Remark: For $x=c x_{0} \in \operatorname{span}\left\{x_{0}\right\}$, we can define $\|x\|_{E}=|c|$.

In order to prove Theorem 1.1, we need some lemmas. Let $X$ be a Hilbert space and $\psi \in C^{1}(X, \mathbf{R})$. As in [17], let $K=\left\{x \in X \mid \psi^{\prime}(x)=\theta\right\}$, $\psi_{m}=\{x \in X \mid \psi(x) \leq m\}$. For an isolated critical point $x_{0}$, the critical group is 
defined by $C_{q}\left(\psi, x_{0}\right)=H_{q}\left(\psi_{c} \cap U,\left(\psi_{c} \backslash\left\{x_{0}\right\}\right) \cap U ; \mathbf{R}\right) \quad$ for $\quad q=0,1,2,3, \cdots$, where $U$ is a neighborhood of $\left\{x_{0}\right\}$ such that $K \cap\left(\psi_{c} \cap U\right)=\left\{x_{0}\right\}$ and $c=\psi\left(x_{0}\right)$.

When $\psi \in C^{2}(X, \mathbf{R})$ and $p \in K$, we have $\psi^{\prime \prime}(p)$ is a self-adjoint operator. We call the dimension of negative space corresponding to the spectral decomposing the Morse index of $p$ and denote it by $m^{-}\left(\psi^{\prime \prime}(p)\right)$, and denote by $m^{0}\left(\psi^{\prime \prime}(p)\right)=\operatorname{dim}\left(\operatorname{ker} \psi^{\prime \prime}(p)\right)$. If $\psi^{\prime \prime}(p)$ has a bounded inverse we say that $p$ is nondegenerate.

From Theorem 3.1 in Chapter 3, Theorem 5.1, 5.2, Corollary 5.2 in Chapter 5 in [17], one can prove the following lemma.

Lemma 2.4. Assume $\psi \in C^{2}(X, \mathbf{R})$ satisfies the (PS) condition, $\psi^{\prime}(\theta)=\theta_{1}$, where $\theta$ is the zero vector in $X$ and $\theta_{1}$ is the zero vector in $X^{*}$ which is the dual space of $X$, and there is a positive integer $\gamma$ such that $\gamma \bar{\epsilon}\left[m^{-}\left(\psi^{\prime \prime}(\theta)\right), m^{-}\left(\psi^{\prime \prime}(\theta)\right)+m^{0}\left(\psi^{\prime \prime}(\theta)\right)\right]$ and $H_{q}\left(X, \psi_{m} ; \mathbf{R}\right)=\delta_{q \gamma} \mathbf{R}$ for some regular $m<\psi(\theta)$, here $\delta_{q \gamma}=\left\{\begin{array}{ll}1 & q=\gamma \\ 0 & q \neq \gamma\end{array}\right.$. Then $\psi$ has a critical point $p_{0} \neq \theta$ with $C_{\gamma}\left(\psi, p_{0}\right) \neq 0$. Moreover, if $\theta$ is a nondegenerate critical point, and $m^{0}\left(\psi^{\prime \prime}\left(p_{0}\right)\right) \leq\left|\gamma-m^{-}\left(\psi^{\prime \prime}(\theta)\right)\right|$, then $\psi$ has another critical point $p_{1} \neq p_{0}, \theta$.

The following lemma is also useful for us to prove the main result.

Lemma 2.5 (Fatou's lemma). Given a measure space $(\Omega, \Sigma, \mu)$ and a set $X \in \Sigma$, let $f_{n}$ be a sequence of $\left(\Sigma, \mathcal{B}_{R_{\geq 0}}\right)$-measurable non-negative functions $f_{n}: X \rightarrow[0,+\infty]$, where $\mathcal{B}_{R_{\geq 0}}$ denotes the $\sigma$-algebra of Borel sets on $[0,+\infty]$. Define the function $f: X \rightarrow[0,+\infty]$ by setting

$$
f(x)=\liminf _{n \rightarrow \infty} f_{n}(x), f(x)=\liminf _{n \rightarrow \infty} f_{n}(x),
$$

for every $x \in X$. Then $f$ is $\left(\Sigma, \mathcal{B}_{R_{\geq 0}}\right)$-measurable, and

$$
\int_{X} f \mathrm{~d} \mu \leq \liminf _{n \rightarrow \infty} \int_{X} f_{n} \mathrm{~d} \mu .
$$

Remark The integrals may be finite or infinite.

\section{Proof of the Main Result}

The proof of Theorem 1.1 will depend on the following lemma.

Lemma 3.1 Under $\left(\mathrm{H}_{1}\right),\left(\mathrm{H}_{2}\right)$, and $\left(\mathrm{H}_{3}\right)$, the functional $\varphi$ satisfies the (PS) condition.

Proof For $\left\{x_{n}\right\} \subset E, \varphi^{\prime}\left(x_{n}\right) \rightarrow \theta$, and $\varphi\left(x_{n}\right)$ is bounded, we shall find a convergent subsequence in $E$. By (1.3), for $u \in E$, we have

$$
\begin{aligned}
\left\langle\varphi^{\prime}\left(x_{n}\right), u\right\rangle= & \int_{0}^{1} x_{n}^{\prime}(t) u^{\prime}(t) \mathrm{d} t-\left(x_{n}(1), u(1)\right) k(\beta)+\left(x_{n}(0), u(0)\right) k(\alpha) \\
& -\int_{0}^{1} f\left(t, x_{n}(t)\right) u(t) \mathrm{d} t .
\end{aligned}
$$

Next, we will prove $\left\{\left\|x_{n}\right\|_{E}\right\}_{1}^{\infty}$ is bounded. Indeed, it suffices to prove that $\left\|x_{n}\right\|_{C}$ is bounded. By a contradiction, we assume that $\left\|x_{n}\right\|_{C} \rightarrow+\infty$, as $n \rightarrow \infty$. Defining 


$$
q_{n}(t)=\left\{\begin{array}{ll}
\frac{f\left(t, x_{n}(t)\right)}{x_{n}(t)} & \left|x_{n}(t)\right| \geq r \\
\lambda(t) & \left|x_{n}(t)\right|<r
\end{array}, \text { and } h_{n}(t)=f\left(t, x_{n}(t)\right)-q_{n}(t) x_{n}(t),\right.
$$

from $\left(\mathrm{H}_{1}\right),\left(\mathrm{H}_{2}\right)$ and $f:[0,1] \times \mathbf{R} \rightarrow \mathbf{R}$ is continuous, we have

$$
a_{0} \leq q_{n}(t) \leq \lambda(t) \text { and }\left|h_{n}(t)\right|<r|\lambda(t)|+C_{0},
$$

where $C_{0}$ is a constant. Then we get

$$
f(t, x(t))=q_{n}(t) x_{n}(t)+h_{n}(t) .
$$

By (3.1), it follows that

$$
\begin{aligned}
\int_{0}^{1}\left(x_{n}^{\prime}(t), u^{\prime}(t)\right) \mathrm{d} t= & \int_{0}^{1}\left(f\left(t, x_{n}(t)\right), u(t)\right) \mathrm{d} t+\left(x_{n}(1), u(1)\right) k(\beta) \\
& -\left(x_{n}(0), u(0)\right) k(\alpha)+\left\langle\varphi^{\prime}\left(x_{n}\right), u\right\rangle .
\end{aligned}
$$

Assuming $y_{n}=\frac{x_{n}}{\left\|x_{n}\right\|_{C}}$, by (3.4), and multiplying $\left\|x_{n}\right\|_{C}^{-1}$ on both sides of (3.5), we can get that

$$
\begin{aligned}
\int_{0}^{1}\left(y_{n}^{\prime}(t), u^{\prime}(t)\right) \mathrm{d} t= & \int_{0}^{1} q_{n}(t) y_{n} u \mathrm{~d} t+\left(y_{n}(1), u(1)\right) k(\beta)-\left(y_{n}(0), u(0)\right) k(\alpha) \\
& +\left\|x_{n}\right\|_{C}^{-1}\left(\int_{0}^{1} h_{n}(t) u(t) \mathrm{d} t+\left\langle\varphi\left(x_{n}\right), u\right\rangle\right) .
\end{aligned}
$$

Furthermore, we add $\int_{0}^{1} y_{n}(t) u(t) \mathrm{d} t$ on two sides of (3.6) to obtain that

$$
\begin{aligned}
\left(y_{n}, u\right)_{E}= & \int_{0}^{1} y^{\prime} u^{\prime} \mathrm{d} t+\int_{0}^{1} y_{n} u \mathrm{~d} t \\
= & \int_{0}^{1} q_{n}(t) y_{n} u \mathrm{~d} t+\int_{0}^{1} y_{n} u \mathrm{~d} t+\left(y_{n}(1), u(1)\right) k(\beta) \\
& -\left(y_{n}(0), u(0)\right) k(\alpha)+\left\|x_{n}\right\|_{C}^{-1}\left(\int_{0}^{1} h_{n}(t) u(t) \mathrm{d} t+\left\langle\varphi\left(x_{n}\right), u\right\rangle\right) .
\end{aligned}
$$

So, by $\left\|y_{n}\right\|_{L^{2}}=\left(\int_{0}^{1} y_{n}^{2}(t) \mathrm{d} t\right)^{\frac{1}{2}} \leq\left\|y_{n}\right\|_{C}=1$ and (3.3) we have

$$
\begin{aligned}
\left\|y_{n}\right\|_{E} & =\sup _{\|u\|_{E} \leq 1}\left(y_{n}, u\right)_{E} \\
& \leq \int_{0}^{1}\left(q_{n}(t) y_{n}(t) u\right) \mathrm{d} t+\int_{0}^{1}\left(y_{n}(t) u\right) \mathrm{d} t+|k(\beta)|+|k(\alpha)|+C_{2} \\
& \leq C_{3}\left\|y_{n}\right\|_{L^{2}}\|u\|_{L^{2}}+|k(\beta)|+|k(\alpha)|+C_{2} \\
& \leq C^{*},
\end{aligned}
$$

where $C_{2}, C_{3}$ and $C^{*}$ are constants. So $\left\{\left\|y_{n}\right\|_{E}\right\}_{1}^{\infty}$ is bounded. Then $\left\{y_{n}\right\}$ has a convergent subsequence. Without loss of generality, we also denoted by $\left\{y_{n}\right\}$. Then $y_{n} \rightarrow y_{0} \quad$ in $E$ and $y_{n} \rightarrow y_{0}$ in $C[0,1]$. By inequality $a_{0}(t) \leq q_{n}(t) \leq \lambda(t)$, we have $q_{n} \rightarrow q_{0}$ in $L^{2}[0,1]$. Then taking the limits on both sides of (3.6), we have, for any $u \in E$,

$$
\begin{aligned}
& \int_{0}^{1} y_{0}^{\prime} u^{\prime} \mathrm{d} t-\left(y_{0}(1), u(1)\right) k(\beta)+\left(y_{0}(0), u(0)\right) k(\alpha)-\int_{0}^{1} q_{0}(t) y_{0} u \mathrm{~d} t=0, \\
& \forall u \in E .
\end{aligned}
$$

From (3.7) and [3], we have that $y_{0}$ is a solution of the following problem: 


$$
\left\{\begin{array}{l}
y^{\prime \prime}(t)+q_{0}(t) y=0, \text { a.e. } t \in[0,1] \\
y(0) \cos \alpha-y^{\prime}(0) \sin \alpha=0 \\
y(1) \cos \beta-y^{\prime}(1) \sin \beta=0
\end{array}\right.
$$

What's more, since $a_{0}(t) \leq q_{n}(t) \leq \lambda(t)$, we have $q_{0}=0$. In fact, by the meaning of the notation " $<$ " and " $\leq$ ", on the one hand, if $q_{0}(t)=\lambda(t)$, then $v\left(q_{0}\right)=v(\lambda)=0$. Therefore, by the definition $v(\cdot)$, this means that (3.8) only has a trivial solution. In fact, by $\left\|y_{n}\right\|_{C}=1$, we obtain $\left\|y_{0}\right\|_{C}=1$. So (3.8) has a nontrivial solution. This is a contradiction. On the other hand, if $a_{0}(t)<q_{0}(t)<\lambda(t)$, then $1=i\left(a_{0}\right)+v\left(a_{0}\right) \leq i\left(q_{0}\right) \leq i(\lambda)=1$ holds. While $y_{0}$ is a nontrivial solution of (3.8), this leads $v\left(q_{0}\right)=1$. So by Proposition 2.1 (4), we get $2=i\left(q_{0}\right)+v\left(q_{0}\right) \leq i(\lambda)=1$. This is also a contradiction. From discussion above, we obtain the conclusion that $q_{0}=a_{0}$. So we immediately get $y_{0} \in \operatorname{span}\left\{x_{0}\right\}$.

Since $y_{0} \in \operatorname{span}\left\{x_{0}\right\}$, there are two cases about $y_{0}$. One is that $y_{0}>0$, the other is that $y_{0}<0$. Without loss of generality, if $y_{0}>0$, we assume $y_{0}=x_{0}$, and if $y_{0}<0$, we assume $y_{0}=-x_{0}$. Firstly, we discuss the situation that $y_{0}>0$. If $y_{0}>0$, i.e. $y_{0}=x_{0}$, then for $\forall \varepsilon>0, \exists N=N(\varepsilon)$ such that for $n>N,\left|y_{n}-x_{0}\right|<\varepsilon$ holds. Here, we take the $\varepsilon$ such that $x_{0}-\varepsilon>0$, i.e. when $n>N,\left\{y_{n}(t)\right\}$ belong to the neighborhood of $y_{0},\left(x_{0}-\varepsilon, x_{0}+\varepsilon\right)$, for all $t \in[0,1]$. This means $y_{n}(t)>x_{0}-\varepsilon$, as $n>N$, for all $t \in[0,1]$.

So by $y_{n}=\frac{x_{n}(t)}{\left\|x_{n}\right\|_{C}}$, we can get that for any $t \in[0,1], \quad x_{n}(t)=y_{n}(t)\left\|x_{n}\right\|_{C}>0$ for $n>N$. Then $x_{n}(t) \rightarrow+\infty$ for all $t \in[0,1]$, as $\left\|x_{n}\right\|_{C} \rightarrow \infty$. By the assumption that $\left\|x_{n}\right\|_{C} \rightarrow \infty$, as $n \rightarrow \infty$, taking the limits on both sides of (3.1) and letting $u=x_{0}$, we can obtain

$$
\begin{aligned}
& \left\langle\varphi^{\prime}\left(x_{n}\right), x_{0}\right\rangle \\
& =\int_{0}^{1} x_{n}^{\prime} x_{0}^{\prime} \mathrm{d} t-\left(x_{n}(1), x_{0}(1)\right) k(\beta)+\left(x_{n}(0), x_{0}(0)\right) k(\alpha)-\int_{0}^{1} f\left(t, x_{n}(t)\right) x_{0} \mathrm{~d} t \\
& =\int_{0}^{1}\left(q_{0}(t) x_{n}-f\left(t, x_{n}\right)\right) x_{0} \mathrm{~d} t \\
& =-\int_{0}^{1} f_{1}\left(t, x_{n}\right) x_{0} \mathrm{~d} t .
\end{aligned}
$$

So, by $\left(\mathrm{H}_{3}\right)$, and (3.9), the following holds

$$
\int_{0}^{1} f_{1}\left(t, x_{n}(t)\right) x_{0} \mathrm{~d} t \rightarrow 0, \text { as } n \rightarrow \infty .
$$

Furthermore, by the Fatou's Lemma and (3.10), we have

$$
\begin{aligned}
0 & =\varliminf_{n \rightarrow \infty} \int_{0}^{1} f_{1}\left(t, x_{n}(t)\right) x_{0} \mathrm{~d} t \geq \int_{0}^{1} \frac{\lim _{n \rightarrow \infty}}{f_{1}}\left(t, x_{n}(t)\right) x_{0} \mathrm{~d} t \\
& =\int_{0}^{1} f_{1}(t,+\infty) x_{0} \mathrm{~d} t,
\end{aligned}
$$

a contradiction to assumption $\left(\mathrm{H}_{3}\right)$. Hence, if $y_{0}>0$, this leads to a contradiction. Secondly, in a similar way, we can show that if $y_{0}<0$, there also be a contradiction. Therefore, the sequence $\left\{\left\|x_{n}\right\|_{C}\right\}_{1}^{\infty}$ is a bounded sequence. By the equality $x_{n}=y_{n}\left\|x_{n}\right\|_{C}$ and the fact that $\left\|y_{n}\right\|_{E}$ is bounded, we can get that 
$\left\{\left\|x_{n}\right\|_{E}\right\}_{1}^{\infty}$ is bounded in $E$. Furthermore, $\left\{x_{n}\right\}_{1}^{\infty}$ has a weak convergent subsequence in $E$, without loss of generality, still denoted by $\left\{x_{n}\right\}_{1}^{\infty}$. So we have $x_{n} \rightarrow x_{*}$ in $E$ and $x_{n} \rightarrow x_{*}$ in $C[0,1]$. In addition, by (3.1), we also have

$$
\begin{aligned}
& \int_{0}^{1} x_{*}^{\prime}(t) u^{\prime}(t) \mathrm{d} t-\left(x_{*}(1), u(1)\right) k(\beta)+\left(x_{*}(0), u(0)\right) k(\alpha) \\
& -\int_{0}^{1}\left(f\left(t, x_{*}(t)\right), u(t)\right) \mathrm{d} t=0 .
\end{aligned}
$$

At last, we only need to finish the mission that $x_{n} \rightarrow x_{*}$ in $E$. Indeed, by (3.5), (3.11) and $x_{n} \rightarrow x_{*}$, we obtain the fact that

$$
\begin{aligned}
\left\|x_{n}-x_{*}\right\|_{E}= & \sup _{\|u\|_{E} \leq 1}\left(x_{n}-x_{*}, u\right)_{E}=\sup _{\|u\|_{E} \leq 1}\left[\int_{0}^{1}\left(x_{n}^{\prime}-x_{*}^{\prime}, u^{\prime}\right) \mathrm{d} t+\int_{0}^{1}\left(x_{n}-x_{*}, u\right) \mathrm{d} t\right] \\
= & \sup _{\|u\|_{E} \leq 1}\left\{\int_{0}^{1}\left(f\left(t, x_{n}\right)-f\left(t, x_{*}\right), u(t)\right) \mathrm{d} t+\left\langle\varphi^{\prime}\left(x_{n}\right)-\varphi^{\prime}\left(x_{*}\right), u\right\rangle\right. \\
& +\left(x_{n}(1)-x_{*}(1), u\right) k(\beta)-\left(x_{*}(0)-x_{n}(0), u\right) k(\alpha) \\
& \left.+\int_{0}^{1}\left(x_{n}-x_{*}, u\right) \mathrm{d} t\right\} \rightarrow 0, \text { as } n \rightarrow \infty .
\end{aligned}
$$

The (PS) condition is verified.

After the preliminary work, we can prove Theorem 1.1.

Proof of Theorem 1.1. Since $v(a) \leq 1$ for any $a \in L^{\infty}[0,1]$, by Lemma 2.4, we only need to prove

$$
H_{q}\left(E, \varphi_{z} ; \mathbf{R}\right) \cong \delta_{q \gamma} \mathbf{R}
$$

for $-z>-\varphi(\theta)$ large enough, where $\gamma=v(0)=i(\lambda)=1$. By Lemma 2.3, we know that $E$ can be split into two subspaces $\operatorname{span}\left\{x_{0}\right\}$ and $E^{+}(\lambda)$, i.e.

$$
E=\operatorname{span}\left\{x_{0}\right\} \oplus E^{+}(\lambda) .
$$

Next, we will take two steps to obtain the proof of (3.12).

First step: For $-z>-\varphi(\theta)$ large enough, we have

$$
H_{q}\left(E, \varphi_{z} ; \mathbf{R}\right) \cong H_{q}\left(\mathcal{M}, \mathcal{M} \cap \varphi_{z} ; \mathbf{R}\right), \quad q=0,1,2, \cdots,
$$

where $\mathcal{M} \subset H$ will be defined later. By assumption, for any $y \in E$, we have

$$
\begin{aligned}
& \left\langle\varphi^{\prime}(x), y\right\rangle \\
& =\int_{0}^{1} x^{\prime} y^{\prime} \mathrm{d} t-(x(1), y(1)) k(\beta)+(x(0), y(0)) k(\alpha)-\int_{0}^{1} f(t, x) y \mathrm{~d} t .
\end{aligned}
$$

We will consider the behavior of $f$ in two subintervals of $[0,1]$. One is $\{t \mid x(t) \geq r\} \subset[0,1]$, the other is $\{t|| x(t) \mid<r\} \subset[0,1]$. Since $f$ is continuous on $[0,1] \times(-\infty,+\infty)$, it is obvious that $f(t, x(t))$ is bounded on $\{t|| x(t) \mid<r\}$. So there exists a constant $M_{1} \in \mathbf{R}$ such that $|f(t, x(t))|<M_{1}$ when $|x(t)|<r$.

By Lemma 2.3, we have a decomposition with respect to $x(t) \in E$, i.e. there exist $x_{+}(t) \in E^{+}(\lambda)$, and $c \in \mathbf{R}$ such that $x=x_{+}+c x_{0}$. When $\left|x_{+}+c x_{0}\right|<r$, we have $\left|c x_{0}\right|<r+\left|x_{+}\right|<r+\left\|x_{+}\right\|_{C}$. Furthermore, we get

$$
\left|c x_{0} \int_{\left|x_{+}+c x_{0}\right|<r} f\left(t, x_{+}+c x_{0}\right) \mathrm{d} t\right|<M_{1}\left(r+\left\|x_{+}\right\|_{C}\right) .
$$

So by (15), we have 


$$
\begin{aligned}
& \int_{\left|x_{+}+c x_{0}\right|<r} f\left(t, x_{+}+c x_{0}\right)\left(x_{+}-c x_{0}\right) \mathrm{d} t \\
= & \int_{\left|x_{+}+x_{0}\right|<r} f\left(t, x_{+}+c x_{0}\right) x_{+} \mathrm{d} t-c x_{0} \int_{\left|x_{+}+c x_{0}\right|<r} f(t, x) \mathrm{d} t \\
\leq & M_{1}\left\|x_{+}\right\|_{C}+M_{1}\left(r+\left\|x_{+}\right\|_{C}\right) .
\end{aligned}
$$

By $\left(\mathrm{H}_{1}\right)$, we have

$$
\begin{aligned}
& \int_{\left|x_{+}+c x_{0}\right| \geq r} f\left(t, x_{+}+c x_{0}\right)\left(x_{+}-c x_{0}\right) \mathrm{d} t \\
= & \int_{\left|x_{+}+c x_{0}\right| \geq r} \frac{f\left(t, x_{+}+c x_{0}\right)}{x_{+}+c x_{0}}\left(x_{+}+c x_{0}\right)\left(x_{+}-c x_{0}\right) \mathrm{d} t \\
= & \int_{\left|x_{+}+c x_{0}\right| \geq r} \frac{f\left(t, x_{+}+c x_{0}\right)}{x_{+}+c x_{0}}\left(x_{+}^{2}-c^{2} x_{0}^{2}\right) \mathrm{d} t \\
= & \int_{\left|x_{+}+c x_{0}\right| \geq r} \frac{f\left(t, x_{+}+c x_{0}\right)}{x_{+}+c x_{0}} x_{+}^{2} \mathrm{~d} t-\int_{\left|x_{+}+c x_{0}\right| \geq r} c^{2} x_{0}^{2} \frac{f(t, x)}{x} \mathrm{~d} t \\
\leq & \int_{\left|x_{+}+c x_{0}\right| \geq r} \lambda(t)\left|x_{+}\right|^{2} \mathrm{~d} t-\int_{\left|x_{+}+c x_{0}\right| \geq r} c^{2} a_{0}(t) x_{0}^{2} \mathrm{~d} t \\
\leq & \int_{0}^{1} \lambda(t)\left|x_{+}\right|^{2} \mathrm{~d} t+M^{\prime},
\end{aligned}
$$

where $M^{\prime}$ is a constant. By (3.14), (3.16), (3.17), Proposition 2.1 (5) and Lemma 2.3 , we obtain

$$
\begin{aligned}
\langle & \left.\varphi^{\prime}(x), x_{+}-c x_{0}\right\rangle \\
= & \int_{0}^{1}\left(x_{+}^{\prime}+c x_{0}^{\prime}, x_{+}^{\prime}-c x_{0}^{\prime}\right) \mathrm{d} t-\left(x_{+}(1)+c x_{0}(1), x_{+}(1)-c x_{0}(1)\right) k(\beta) \\
& +\left(x_{+}(0)+c x_{0}(0), x_{+}(0)-c x_{0}(0)\right) k(\alpha)-\int_{0}^{1} f\left(t, x_{+}+c x_{0}\right)\left(x_{+}-c x_{0}\right) \mathrm{d} t \\
= & \int_{0}^{1}\left|x_{+}^{\prime}\right|^{2} \mathrm{~d} t-\left(x_{+}(1), x_{+}(1)\right) k(\beta)+\left(x_{+}(0), x_{+}(0)\right) k(\alpha) \\
& -c^{2}\left[\int_{0}^{1}\left|x_{0}^{\prime}\right|^{2} \mathrm{~d} t-\left(x_{0}(1), x_{0}(1)\right) k(\beta)+\left(x_{0}(0), x_{0}(0)\right) k(\alpha)\right] \\
& -\left\{\int_{\left|x_{+}+c x_{0}\right| \geq r} f\left(t, x_{+}+c x_{0}\right)\left(x_{+}-c x_{0}\right) \mathrm{d} t+\int_{\left|x_{+} c x_{0}\right|<r} f\left(t, x_{+}+c x_{0}\right)\left(x_{+}-c x_{0}\right) \mathrm{d} t\right\} \\
\geq & \int_{0}^{1}\left|x_{+}^{\prime}\right|^{2} \mathrm{~d} t-\left(x_{+}(1), x_{+}(1)\right) k(\beta)+\left(x_{+}(0), x_{+}(0)\right) k(\alpha) \\
& -\int_{0}^{1} c^{2} a_{0}(t) x_{0}^{2} \mathrm{~d} t-\int_{0}^{1} \lambda(t) x_{+}^{2} \mathrm{~d} t-M^{\prime}-M_{1}\left\|x_{+}\right\|_{C}-M_{1}\left(r+\left\|x_{+}\right\|_{C}\right) \\
= & q_{\lambda}\left(x_{+}, x_{+}\right)-2 M_{1}\left\|x_{+}\right\|_{C}-r M_{1}-\int_{0}^{1} c^{2} a_{0}(t) x_{0}^{2} \mathrm{~d} t-M^{\prime} \\
\geq & C_{1}\left\|x_{+}\right\|_{E}^{2}-C_{4}\left\|x_{+}\right\|_{E}-r M_{1}+M_{0},
\end{aligned}
$$

where $C_{1}(>0), C_{4}(>0)$ and $M_{0}$ are constants. And hence, there exists $R_{0}>0$ such that

$$
\left\langle\varphi^{\prime}(x), x_{+}-c x_{0}\right\rangle>1, \forall x \in E \text { with }\left\|x_{+}\right\|_{E}>R_{0} .
$$

Set $\mathcal{M}=\left(E^{+}(\lambda) \cap B_{R_{0}}\right) \oplus \operatorname{span}\left\{x_{0}\right\}$, where $B_{R_{0}}=\left\{x \in E \mid\|x\|_{E} \leq R_{0}\right\}$. We want to define a deformation from $\left(E, \varphi_{z}\right)$ to $\left(\mathcal{M}, \mathcal{M} \cap \varphi_{z}\right)$. Since for every $x=x_{+}+c x_{0} \in \mathcal{M}, f$ is decreasing along vector field $V(x)=-x_{+}+c x_{0}$, we can 
define the flow $\sigma=\sigma(t, x)=\mathrm{e}^{-t} x_{+}+\mathrm{e}^{t} c x_{0}$ and $T_{x}=\ln \left(\left\|x_{+}\right\|_{E}\right)-\ln R_{0}$, which is the first time that $\sigma(t, x)$ arrives at $\mathcal{M}$. Then the deformation is

$$
\eta\left(t, x_{+}+c x_{0}\right)= \begin{cases}x_{+}+c x_{0}, & \left\|x_{+}\right\|_{E} \leq R_{0}, \\ \sigma\left(T_{x} t, x\right), & \left\|x_{+}\right\|_{E}>R_{0} .\end{cases}
$$

One can verify that $\eta:[0,1] \times E \rightarrow E$ is continuous and satisfy

$$
\begin{aligned}
& \eta(0, \cdot)=i d_{E}, \eta(1, E) \subset \mathcal{M}, \eta\left(1, \varphi_{z}\right) \subset \mathcal{M} \cap \varphi_{z}, \\
& \eta\left(t, \varphi_{z}\right) \subset \varphi_{z},\left.\eta(t, \cdot)\right|_{\mathcal{M}}=i d_{\mathcal{M}} \quad \forall t \in[0,1] .
\end{aligned}
$$

Then, $\left(\mathcal{M}, \mathcal{M} \cap \varphi_{z}\right)$ is a deformation retract of $\left(E, \varphi_{z}\right)$. So (3.13) is verified.

Second step: we will prove the following

$$
H_{q}\left(\mathcal{M}, \mathcal{M} \cap \varphi_{z} ; \mathbf{R}\right) \cong \delta_{q \gamma} \mathbf{R},
$$

for any $-z>-\varphi(\theta)$ large enough. In fact, assuming that $|x(t)|=\left|x_{+}+c x_{0}\right| \geq r$, by $\left(\mathrm{H}_{1}\right)$, we will have two cases: one is $f_{1}(t, x) \geq 0$ as $x \geq r$, another is $f_{1}(t, x) \leq 0$ as $x \leq-r$. Firstly, we analyze the situation that $f_{1}(t, x) \geq 0$ as $x=x_{+}+c x_{0} \geq r$. Since $f_{1}(t,+\infty) x_{0}=\liminf _{x \rightarrow+\infty} f_{1}(t, x) x_{0}=\liminf _{x \rightarrow+\infty} f_{1}(t, y) x_{0}$ and $\inf _{y \geq x} f_{1}(t, y) x_{0}$ is a monotonically increasing nonnegative function with respect to $x \geq r>0$, by $\left(\mathrm{H}_{3}\right)$, we have

$$
\int_{0}^{1} f_{1}(t, \infty) x_{0} \mathrm{~d} t=\int_{0}^{1} \liminf _{x \rightarrow+\infty} f_{y \geq x}(t, y) x_{0} \mathrm{~d} t=\lim _{x \rightarrow+\infty} \int_{0}^{1} \inf _{y \geq x} f_{1}(t, y) x_{0} \mathrm{~d} t>0 .
$$

Then $\exists x^{*}(\geq r) \in \mathbf{R}$, for all $l>x^{*}, \int_{0}^{1} \inf _{y \geq l} f_{1}(t, y) x_{0} \mathrm{~d} t>0$ holds. What's more, since $x_{0}(t)>0$ for all $t \in[0,1]$. So there exists a $M:=\sup _{t \in[0,1]}\left|x_{0}(t)\right|>0$, such that $M \int_{0}^{1} f_{1}(t, y) \mathrm{d} t \geq \int_{0}^{1} \inf _{y \geq l} f_{1}(t, y) x_{0} \mathrm{~d} t>0$, i.e. $\int_{0}^{1} \inf _{y \geq l} f_{1}(t, y) \mathrm{d} t>0$.

So letting $x=x_{+}+c x_{0}>l$, where $c \in \mathbf{R}, l$ is fixed and $l>x^{*} \geq r$, we have

$$
\begin{aligned}
F(t, x) & =\int_{0}^{x} f(t, s) \mathrm{d} s=\int_{0}^{x_{+}+c x_{0}}\left[f(t, s)-a_{0}(t) s\right] \mathrm{d} s+\frac{1}{2} a_{0}(t)\left(x_{+}+c x_{0}\right)^{2} \\
& =\int_{0}^{l}\left[f(t, s)-a_{0}(t) s\right] \mathrm{d} s+\int_{l}^{x_{+}+c x_{0}}\left[f(t, s)-a_{0}(t) s\right] \mathrm{d} t+\frac{1}{2} a_{0}(t) x^{2} \\
& \geq M_{4}+\left(x_{+}+c x_{0}-l\right) \inf _{x_{+}+c x_{0} \geq y \geq l} f_{1}(t, y)+\frac{1}{2} a_{0}(t) x^{2} \\
& \geq M_{4}+\left(x_{+}+c x_{0}-l\right) \inf _{y \geq l} f_{1}(t, y)+\frac{1}{2} a_{0}(t) x^{2} .
\end{aligned}
$$

Furthermore, by (3.19), we obtain

$$
\begin{aligned}
\varphi(x)= & \frac{1}{2}\left[\int_{0}^{1}\left|x^{\prime}\right|^{2} \mathrm{~d} t-(x(1), x(1)) k(\beta)+(x(0), x(0)) k(\alpha)\right]-\int_{0}^{1} F(t, x) \mathrm{d} t \\
= & \frac{1}{2}\left[\int_{0}^{1}\left(x_{+}^{\prime 2}+2 c x_{+}^{\prime} x_{0}^{\prime}+c^{2} x_{0}^{\prime 2}\right) \mathrm{d} t-\left(x_{+}(1)+c x_{0}(1)\right)^{2} k(\beta)\right. \\
& \left.+\left(x_{+}(0)+c x_{0}(0)\right)^{2} k(\alpha)\right]-\int_{0}^{1} F(t, x) \mathrm{d} t
\end{aligned}
$$




$$
\begin{aligned}
& \leq \frac{1}{2} \int_{0}^{1}\left(x_{+}^{\prime 2}+2 c x_{+}^{\prime} x_{0}^{\prime}+c^{2} x_{0}^{\prime 2}\right) \mathrm{d} t-x_{+}(1)^{2} k(\beta)+x_{+}(0)^{2} k(\alpha) \\
& -\frac{1}{2} \int_{0}^{1} a_{0}(t)\left(x_{+}^{2}+2 c x_{+} x_{0}+c^{2} x_{0}^{2}\right) \mathrm{d} t-\frac{1}{2} c^{2} x_{0}(1)^{2} k(\beta)+\frac{1}{2} c^{2} x_{0}(0)^{2} k(\alpha) \\
& -\frac{1}{2} c x_{+}(1) x_{0}(1) k(\beta)+\frac{1}{2} c x_{+}(0) x_{0}(0) k(\alpha) \\
& -M_{4}-\left(x_{+}+c x_{0}-l\right) \int_{0}^{1} \inf _{y \geq l} f_{1}(t, y) \mathrm{d} t \\
& =\frac{1}{2}\left[\int_{0}^{1} x_{+}^{\prime 2}-a_{0}(t) x_{+}^{2} \mathrm{~d} t-x_{+}(1)^{2} k(\beta)+x_{+}(0)^{2} k(\alpha)\right] \\
& +c \int_{0}^{1} x_{+}^{\prime} x_{0}^{\prime}-a_{0}(t) x_{+} x_{0} \mathrm{~d} t-c x_{+}(1) x_{0}(1) k(\beta)+c x_{+}(0) x_{0}(0) k(\alpha) \\
& +\frac{c^{2}}{2}\left[\int_{0}^{1}\left(x_{0}^{\prime 2}-a_{0}(t) x_{0}^{2}\right) \mathrm{d} t-x_{0}(1)^{2} k(\beta)+x_{0}(0)^{2} k(\alpha)\right] \\
& -M_{4}-\left(x_{+}+c x_{0}-l\right) \int_{0}^{1} \inf _{y \geq l} f_{1}(t, y) \mathrm{d} t \\
& \quad=\frac{1}{2} \int_{0}^{1}\left(x_{+}^{\prime 2}-a_{0}(t) x_{+}^{2}\right) \mathrm{d} t-x_{+}(1)^{2} k(\beta)+x_{+}(0)^{2} k(\alpha) \\
& \quad-M_{4}-\left(x_{+}+c x_{0}-l\right) \int_{0}^{1} \inf f_{1}(t, y) \mathrm{d} t .
\end{aligned}
$$

So we get $\varphi(x) \rightarrow-\infty$, as $c \rightarrow+\infty$, uniformly in $x_{+} \in E^{+}(\lambda) \cap B_{R_{0}}$. Secondly, we analyze the situation that $f_{1}(t, x) \leq 0$ as $x=x_{+}+c x_{0} \leq-r$. In a similar way, we also get $\varphi(x) \rightarrow-\infty$, as $c \rightarrow-\infty$, uniformly in $x_{+} \in E^{+}(\lambda) \cap B_{R_{0}}$. So we obtain that

$$
\varphi(x) \rightarrow-\infty \Leftrightarrow|c| \rightarrow+\infty \text { uniformly in } x_{+} \in E^{+}(\lambda) \bigcap B_{R_{0}} .
$$

Thus, there exist $T>0, z_{1}<z_{2}<-T, R_{1}>R_{2}>R_{0}$ such that

$$
\begin{aligned}
& \left(E^{+}(\lambda) \cap B_{R_{0}}\right) \oplus\left(\operatorname{span}\left\{x_{0}\right\} \backslash B_{R_{1}}^{\prime}\right) \subset \varphi_{z_{1}} \cap \mathcal{M} \\
& \subset\left(E^{+}(\lambda) \cap B_{R_{0}}\right) \oplus\left(\operatorname{span}\left\{x_{0}\right\} \backslash B_{R_{2}}^{\prime}\right) \subset \varphi_{z_{2}} \cap \mathcal{M},
\end{aligned}
$$

where $B_{R}^{\prime}=\left\{c x_{0} \mid c \in \mathbf{R}\right.$ and $\left.\left\|c x_{0}\right\|_{E}=|c| \leq R\right\}$ by remark. For the sake of convenience, we set $\mathcal{N}_{R}=\left(E^{+}(\lambda) \cup B_{R_{0}}\right) \oplus\left(\operatorname{span}\left\{x_{0}\right\} \backslash B_{R}^{\prime}\right)$. Then (3.20) can also be denoted as

$$
\mathcal{N}_{R_{1}} \subset \varphi_{z_{1}} \cap \mathcal{M} \subset \mathcal{N}_{R_{2}} \subset \varphi_{z_{2}} \cap \mathcal{M}
$$

We now begin to define a deformation from $\mathcal{M} \cap \varphi_{z_{2}}$ to $\mathcal{M} \cap \varphi_{z_{1}}$. For every $x \in \mathcal{M} \cap\left(\varphi_{z_{2}} \backslash \varphi_{z_{1}}\right)$, since the flow is defined by $\sigma(t, x)=\mathrm{e}^{-t} x_{+}+\mathrm{e}^{t} c x_{0}$, $\varphi(\sigma(t, x))$ is continuous with respect to $t, \varphi(\sigma(0, x))=\varphi(x)>z_{1}$ and $\varphi(\sigma(t, x)) \rightarrow-\infty$ as $t \rightarrow+\infty$, so the time $t=T_{1}(x)$ arriving at $\varphi_{z_{1}} \cap \mathcal{M}$ exists uniquely and is defined by $\varphi(\sigma(t, x))=z_{1}$. Since

$$
\begin{aligned}
\frac{\mathrm{d} \varphi(\sigma(t, x))}{\mathrm{d} t} & =\left\langle\varphi^{\prime}(\sigma(t, x)), \sigma^{\prime}(t, x)\right\rangle \\
& =\left\langle\varphi^{\prime}\left(\mathrm{e}^{-t} x_{+}+\mathrm{e}^{t} c x_{0}\right),-\mathrm{e}^{-t} x_{+}+\mathrm{e}^{t} c x_{0}\right\rangle \leq-1
\end{aligned}
$$

as $t>0$, the continuity of $t=T_{1}(x)$ comes from the implicit function theorem. 
Define

$$
\begin{aligned}
\eta_{1}(t, x) & =x, \quad \forall x \in \varphi_{z_{1}} \cap \mathcal{M} \\
& =\sigma\left(T_{1}(x) t, x\right), \quad \forall x \in \mathcal{M} \cap\left(\varphi_{z_{2}} \backslash \varphi_{z_{1}}\right)
\end{aligned}
$$

then $\eta_{1}:[0,1] \times \varphi_{z_{2}} \cap \mathcal{M} \rightarrow \varphi_{z_{2}} \cap \mathcal{M}$ is continuous, and is a deformation from $\varphi_{z_{2}} \cap \mathcal{M}$ to $\varphi_{z_{1}} \cap \mathcal{M}$ and $\tau_{1}=\eta(1,(\cdot)): \varphi_{z_{2}} \cap \mathcal{M} \rightarrow \varphi_{z_{1}} \cap \mathcal{M}$ is a strong deformation retract. Hence,

$$
H_{q}\left(\varphi_{z_{2}} \cap \mathcal{M}, \varphi_{z_{1}} \cap \mathcal{M} ; \mathbf{R}\right) \cong 0 .
$$

Recall that for any topological spaces $Z \subseteq Y \subseteq X$, we have exact sequences

$$
H_{q}(Y, Z ; \mathbf{R}) \rightarrow H_{q}(X, Z ; \mathbf{R}) \rightarrow H_{q}(X, Y ; \mathbf{R}) \rightarrow H_{q-1}(Y, Z ; \mathbf{R}) .
$$

From (3.20), in order to prove

$$
H_{q}\left(\mathcal{M}, \varphi_{z_{2}} \cap \mathcal{M} ; \mathbf{R}\right) \cong H_{q}\left(\mathcal{M}, \mathcal{N}_{R_{1}} ; \mathbf{R}\right)
$$

we only to prove

$$
H_{q}\left(\varphi_{z_{2}} \cap \mathcal{M}, \mathcal{N}_{R_{1}} ; \mathbf{R}\right) \cong 0
$$

And from (3.21), it suffices to verify

$$
H_{q}\left(\varphi_{z_{1}} \cap \mathcal{M}, \mathcal{N}_{R_{1}} ; \mathbf{R}\right) \cong 0 .
$$

Let $\tau_{2}:[0,1] \times \mathcal{N}_{R_{2}} \rightarrow \mathcal{N}_{R_{2}}$ satisfy

$$
\begin{aligned}
\tau_{2}\left(t, x_{+}+c x_{0}\right) & =x_{+}+c x_{0}, \quad|c|>R_{1}, \\
& =x_{+}+\frac{c}{|c|}\left(t R_{1}+(1-t) c\right) x_{0}, \quad R_{2}<|c| \leq R_{1} .
\end{aligned}
$$

We can verify that $\tau:=\tau_{1} \circ \tau_{2}:[0,1] \times \varphi_{z_{1}} \cap \mathcal{M} \rightarrow \varphi_{z_{1}} \cap \mathcal{M}$ is continuous, where $\tau_{1} \circ \tau_{2}(t, x)=\tau_{1}\left(\tau_{2}(t, x)\right), t \in[0,1], x \in \varphi_{z_{1}} \cap \mathcal{M}$, and satisfies

$$
\tau[0, x]=\eta_{1}\left(1, \tau_{2}(0, x)\right)=\eta_{1}\left(1, x_{+}+c x_{0}\right)=x_{+}+c x_{0}=x
$$

for any $x \in \varphi_{z_{1}} \cap \mathcal{M}$. So $\tau[0, \cdot]=i d_{\varphi_{z_{1}} \cap \mathcal{M}}$. And

$$
\tau\left[t, x_{+}+c x_{0}\right]=\tau_{1} \circ \tau_{2}[t, x]=\eta_{1}(1, x)=x,
$$

for any $x \in \mathcal{N}_{R_{1}}$. So $\left.\tau[t, \cdot]\right|_{\mathcal{N}_{R_{1}}}=i d_{\mathcal{N}_{R_{1}}}$. We can also see that $\tau$ satisfy $\tau\left(1, \varphi_{z_{1}} \cap \mathcal{M}\right) \subset \mathcal{N}_{R_{1}}, \tau\left(1, \mathcal{N}_{R_{1}}\right) \subset \mathcal{N}_{R_{1}}, \tau\left(t, \mathcal{N}_{R_{1}}\right) \subset \mathcal{N}_{R_{1}}$. Then $\left(\mathcal{N}_{R_{1}}, \mathcal{N}_{R_{1}}\right)$ is a deformation retract of $\left(\varphi_{z_{1}} \cap \mathcal{M}, \mathcal{N}_{R_{1}}\right)$. This means (3.22) and hence (3.21) holds. Finally from (3.21) we have

$$
\begin{aligned}
& H_{q}\left(\mathcal{M}, \mathcal{M} \cap \varphi_{z_{2}} ; \mathbf{R}\right) \\
& \cong H_{q}\left(\left(E^{+}(\lambda) \cap B_{R_{0}}\right) \oplus \operatorname{span}\left\{x_{0}\right\},\left(E^{+}(\lambda) \cap B_{R_{0}}\right) \oplus\left(\operatorname{span}\left\{x_{0}\right\} \backslash B_{R_{1}}^{\prime}\right) ; \mathbf{R}\right) \\
& \cong H_{q}\left(\operatorname{span}\left\{x_{0}\right\} \cap B_{R_{1}}^{\prime} ; \partial\left(\operatorname{span}\left\{x_{0}\right\} \cap B_{R_{1}}^{\prime}\right) ; \mathbf{R}\right) \\
& =\delta_{q \gamma} \mathbf{R}, \quad q=0,1,2,3, \cdots .
\end{aligned}
$$

Here in the second $\cong$ we used the deformation $\zeta:[0,1] \times \mathcal{M} \rightarrow \mathcal{M}$ defined by $\zeta(t, x)=t x_{+}+c x_{0}$, and excision property. So (3.18) is proved. And by (3.13) and (3.18), (3.12) is obtained. The proof is completed. 
In our theorem, we get one nontrivial solution of Equations (1.1)-(1.3). By adding assumption $\left(\mathrm{H}_{4}\right)$, we get two nontrivial solutions of Equations (1.1)-(1.3).

\section{Conclusion}

By index theories established in this paper, and Morse theory, we study the functional corresponding to the problem to obtain more nontrivial solutions of asymptotically linear ordinary differential equations satisfying Sturm-Liouville BVPs with resonance. It's better than the results obtained by topological degree method.

\section{Acknowledgements}

The authors would like to express their sincere thanks to the editors and reviewers for their remarkable comments, suggestions, and ideas that helped to improve this paper.

\section{Funding}

This research work was partially supported by the National Science Foundation of China (11501178).

\section{Conflicts of Interest}

The authors declare that they have no competing interests.

\section{References}

[1] Dong, Y. (2000) On Equivalent Conditions for the Solvability of Equation $\left(p(t) x^{\prime}\right)^{\prime}+f(t, x)=h(t)$ Satisfying Linear Boundary Conditions with $f$ Restricted by Linear Growth Conditions. Journal of Mathematical Analysis and Applications, 245, 204-220. https://doi.org/10.1006/jmaa.2000.6752

[2] Mawhin, J. and Willem, M. (1989) Critical Point Theory and Hamiltonian System. In: John, F., Marsden, J.E. and Sirovich, L., Eds., Applied Mathematical Sciences, Springer, Berlin, 153-166. https://doi.org/10.1007/978-1-4757-2061-7

[3] Dong, Y. (2010) Index for Linear Selfadjoint Operator Equations and Nontrivial Solutions for Asymptotically Linear Operator Equations. Calculus of Variations and Partial Differential Equations, 38, 75-109. https://doi.org/10.1007/s00526-009-0279-5

[4] Conley, C. and Zehnder, E. (1984) Morse-Type Index Theory for Flows and Periodic Solutions for Hamiltonian Equations. Communications on Pure and Applied Mathematics, 37, 207-253. https://doi.org/10.1002/cpa.3160370204

[5] Long, Y. (1990) Maslov-Type Index Theory, Degenerate Critical Points, and Asymptotically Linear Hamiltonian Systems. Science in China, 33, 1409-1419.

[6] Long, Y. (1997) A Maslov-Type Index Theory for Symplectic Paths. Topological Methods in Nonlinear Analysis, 10, 47-78. https://doi.org/10.12775/TMNA.1997.021

[7] Long, Y. and Zehnder, E. (1990) Morse Theory for Forced Oscillations of Asymptotically Linear Hamiltonian Systems. In: Alberverio, S., et al., Eds., Stochastic 
Processes, Phiscs and Geometry, World Scientific, Teaneck, 528-563.

[8] Ekeland, I. (1990) Convexity Methods in Hamiltonian Mechanics. In: Bombieri, E., Feferman, S., et al., Eds., Ergebnisse der Mathematik und ihrer Grenzgebiete, Springer, Berlin, 110-186. https://doi.org/10.1007/978-3-642-74331-3

[9] Long, Y. (2002) Index Theory for Symplectic Paths with Applications. In: Chambert-Loir, A., Lu, J.-H., Ruzhansky, M. and Tschinkel, Y., Eds., Progress in Mathematics, Birkhäuser, Basel, 132-173. https://doi.org/10.1007/978-3-0348-8175-3

[10] Li, S. and Liu, J. (1989) Morse Theory and Asymptotic Linear Hamiltonian System. Journal of Differential Equations, 78, 53-73. https://doi.org/10.1016/0022-0396(89)90075-2

[11] Dong, Y. (2005) Index Theory, Nontrivial Solutions, and Asymptotically Linear Second-Order Hamiltonian Systems. Journal of Differential Equations, 214, 233-255. https://doi.org/10.1016/j.jde.2004.10.030

[12] Dong, D. and Long, Y. (1997) The Iteration Formula of Maslov-Type Theory Applications to Nonliear Haniltonian Systems. Transactions of the American Mathematical Society, 349, 2619-2661. https://doi.org/10.1090/S0002-9947-97-01718-2

[13] Ekeland, I. and Hofer, H. (1985) Periodic Solutions with Prescribed Periodic for Convex Autonomous Hamilyonian Systems. Inventiones Mathematicae, 81, 155-188. https://doi.org/10.1007/BF01388776

[14] Leach, D.E. (1970) On Poincaré's Perturbation Theorem and a Theorem of W.S. Loud. Journal of Differential Equations, 7, 34-53.

https://doi.org/10.1016/0022-0396(70)90122-1

[15] Fabry, C. (1995) Landesman-Lazer Conditions for Periodic Boundary Value Problems with Asymmetric Nonliearities. Journal of Differential Equations, 116, 405-418. https://doi.org/10.1006/jdeq.1995.1040

[16] Iannacci, R. and Nkashama, M.N. (1987) Unbounded Perturbations of Forced Second Order Ordinary Differential Equations at Resonance. Journal of Differential Equations, 69, 289-309. https://doi.org/10.1016/0022-0396(87)90121-5

[17] Chang, K.C. (1993) Infinite Dimensinal Morse Theory and Mutiple Solition Problems. In: Brezis, H., Ed., Progress in Nonlinear Differential Equations and Their Applications, Birkhauser, Basel, 179-228.

https://doi.org/10.1007/978-1-4612-0385-8

[18] Fabry, C. and Fonda, A. (1990) Periodic Solutions of Nonlinear Differential Equations with Double Resonance. Annali di Matematica Pura ed Applicata, 157, 99-116. https://doi.org/10.1007/BF01765314

[19] Shan, Y. (2011) Multiple Solutions of Generalized Asymptotical Linear Hamiltonian Systems Satisfying Sturm-Liouville Boundary Conditions. Nonlinear Analysis. Theory, Methods and Applications, 74, 4809-4819. https://doi.org/10.1016/j.na.2011.04.053

[20] Li, K. (2011) Multiple Solutions for an Asymptotically Linear Duffing Equations with Neumann Boundary Value Conditions. Nonlinear Analysis. Theory, Methods and Applications, 74, 2819-2830. https://doi.org/10.1016/j.na.2011.01.003

[21] Li, K., Li, J. and Mao, W. (2013) Multiple Solutions for Asymptotically Linear Duffing Equations with Neumann Boundary Value Conditions (II). Journal of Mathematical Analysis and Applications, 401, 548-553.

https://doi.org/10.1016/j.jmaa.2012.12.003 